\title{
Causal Association of Trauma With Subsequent Psychiatric Disorder: A Mendelian Randomization Study
}

\author{
Dongqing Gu \\ Army Medical University \\ Shan Ou \\ First People's Hospital of Chengdu \\ Guodong Liu ( $\nabla$ frankliugd@163.com ) \\ Army Medical University
}

\section{Research Article}

Keywords: trauma, psychiatric disorder, depression, Mendelian randomization, causal association

Posted Date: September 15th, 2021

DOI: https://doi.org/10.21203/rs.3.rs-870584/v1

License: (c) (i) This work is licensed under a Creative Commons Attribution 4.0 International License. Read Full License 


\section{Abstract \\ Objective}

Trauma has been proposed as a risk factor for the development of psychiatric disorder. This study aimed to determine the causal association between them.

\section{Methods}

Two-sample Mendelian randomization analyses were performed to estimate the causal association between trauma and psychiatric disorder. We obtained summary-level data for genetic variants associated with trauma and the corresponding association with psychiatric disorder from previous genome-wide association studies, and inverse variance weighted was used as the main method in our Mendelian randomization analysis.

\section{Results}

Genetically predisposed trauma was associated with an increased risk of psychiatric disorder (odds ratio [OR $]=1.02$, 95\% confidence interval $[\mathrm{Cl}], 1.01-1.02$,), mood disorder $(\mathrm{OR}=1.01,95 \% \mathrm{Cl}, 1.00-1.01)$ and depression $(\mathrm{OR}=1.02,95 \%$ $\mathrm{Cl}, 1.01-1.02)$ in UK Biobank, as well as increased risk of mood disorder (OR $=1.23,95 \% \mathrm{Cl}, 1.03-1.48)$, depression (OR $=1.10,95 \% \mathrm{Cl}, 1.04-1.17)$, bipolar disorder $(\mathrm{OR}=1.24,95 \% \mathrm{Cl}, 1.04-1.49)$ and schizophrenia $(\mathrm{OR}=1.47,95 \% \mathrm{Cl}, 1.21-$ 1.78 ) in data source from MR Base. However, Mendelian randomization evidence did not support an association between trauma and risk of post-traumatic stress disorder, anxiety disorder, sleep disorder, and eating disorder.

\section{Conclusions}

Findings from our Mendelian randomization analysis suggested that trauma might be causally associated with an increased risk of some common psychiatric disorder such as depression.

\section{Introduction}

An estimated $89 \%$ of city dwellers have experienced traumatic events ${ }^{1}$. Experiencing traumatic events such as exposure to childhood physical, sexual, and emotional abuse; partner-based physical, sexual, and emotional abuse and other traumatic events, including exposure to sexual assault, violent crime, life-threatening accident, and witnessing violent death may contribute to psychiatric disorder ${ }^{2-7}$. However, whether their relationships are causality remains unclear since the results of observational studies are usually interfered by recall and reporting bias as they are susceptible to confounding factors ${ }^{8}$. Randomized clinical trials (RCTs) are arguably the gold standard to infer causality, however, such trials might be prohibitively costly, require the consideration of ethics, or be otherwise unfeasible.

A complement to traditional epidemiology is to conduct Mendelian randomization (MR) analysis. This approach utilizes genetic variants as a proxy for an exposure of interest to investigate its potential to have a causal association with a disorder $^{9}$. As the inheritance of genetic variants is randomized at meiosis, the association between the alleles and the outcome are unlikely to be affected by reverse causality and confounding factors ${ }^{10}$. Importantly, the available studies have demonstrated that reported trauma exposure is heritable, with twin heritability estimates of $20-50 \%{ }^{11,12}$, and single nucleotide polymorphisms (SNPs)-based heritability estimates of $30 \%{ }^{13}$. Recently, a genome-wide association 
study (GWAS) reported that SNPs were associated with trauma, making it possible to explore the causal relationship between trauma and psychiatric disorder using a two-sample Mendelian randomization analysis ${ }^{5}$.

In this study, we used a two-sample Mendelian randomization approach to comprehensively estimate genetically predisposed trauma and risk of psychiatric disorder using summary data from the Gene ATLAS and the MR-Base platform. We aim to shed light on whether trauma was a risk factor of psychiatric disorder.

\section{Materials And Methods}

\section{Selection of genetic variants}

Instrumental variables for trauma were derived from a recent GWAS including 35,269 European ancestry cases, and 63,451 European ancestry controls ${ }^{5}$. SNPs associated with trauma were selected at the $P$-value $<5 \times 10^{-6}$, since only several SNPs arrived at a genome-wide significance level $\left(P<5 \times 10^{-8}\right)$. After extracting the summary data for the SNPS, we pruned all SNPs in linkage disequilibrium (LD) using an $r^{2}$ threshold $<0.1$, and selected the SNPs with the lowest Pvalue as an independent instrument, resulting in a total of 73 independent SNPs associated with trauma. We calculated the proportion of variance $\left(\mathrm{R}^{2}\right)$ explained in the risk factor by the $\mathrm{SNP}(\mathrm{s})$ and the strength of the instrument (F-statistic) using the formulae from Yarmolinsky et al. ${ }^{14}$. The variance explained by the independent variants was $1.79 \%$, and the F-statistic was 25 , satisfying the threshold of $>10^{15}$. Detailed information of the SNPs used as instrumental variables was displayed in Supplementary Table 1.

\section{Outcome source}

Summary statistics for the genetic association estimates of each SNP used as instrumental variables with psychiatric disorder were obtained from the Gene ATLAS (geneatlas.roslin.ed.ac.uk) and MR-Base platform (www.mrbase.org). Gene ATLAS is a large database of associations between hundreds of traits and millions of variants using the UK Biobank cohort, and MR Base database holds a collection of the summary statistics from a large number of GWASs.

\section{Mendelian randomization analysis}

The inverse variance weighted (IVW) method was used to estimate the effect of the exposure on the outcome from the slope of the relationship between bXG (SNP-exposure association) and bYG (SNP-outcome association).Several sensitivity analyses including simple mode, weighted mode, MR-Egger, and weighted median method were used to evaluate the robustness of our main analyses. To further assess the potential presence of horizontal pleiotropy, we used Cochran's Q for heterogeneity and the intercept from the MR-Egger method ${ }^{16,17}$. Leave-one-out analyse was employed to test the sensitivity of our results to single SNP effects.

\section{Statistical analysis}

All analyses were conducted using the package TwoSampleMR (version 0.5.6) in R (version 3.6.3), and the TwoSampleMR R package curated by MR-Base. All tests were two-sided, and $P$ value of less than 0.05 was considered statistically significant unless stated otherwise.

\section{Results}

Genetically predisposed trauma was associated with overall psychiatric disorder risk in the UK Biobank (odds ratio [OR] $=1.02,95 \%$ confidence interval [CI], 1.01-1.02, $P=2.32 \mathrm{E}-07$ ). Although the Egger intercept did not indicate the presence of horizontal pleiotropy, significant heterogeneity (Cochran's $Q P=0.01$ ) was observed for the inverse-variance weighted 
estimate. We had consistent findings using two other Mendelian randomization analysis methods (weighted median and weighted mode). However, using summary statistics from MR Base, we showed no association between genetically predisposed trauma and overall psychiatric disorder risk. See Table 1, Supplementary Table S2, and Supplementary Table S3. 
Table 1

Mendelian randomization analyses of the causality between genetically predisposed trauma and psychiatric disorder risk.

\begin{tabular}{|c|c|c|c|c|c|c|c|c|}
\hline Outcome & $\begin{array}{l}\text { Outcome } \\
\text { source }\end{array}$ & Consortium & $\begin{array}{l}\text { Sample } \\
\text { size }\end{array}$ & $\begin{array}{l}\text { SNPs } \\
(N)\end{array}$ & $\begin{array}{l}\text { OR } \\
(95 \% \\
\text { Cl) }\end{array}$ & $P$ value & $\begin{array}{l}\text { Heterogeneity } \\
Q \text { tests } P \\
\text { value }\end{array}$ & $\begin{array}{l}\text { MR- } \\
\text { Egger } \\
\text { intercept } \\
\text { test } P \\
\text { value }\end{array}$ \\
\hline \multirow[t]{2}{*}{$\begin{array}{l}\text { Psychiatric } \\
\text { disorder }\end{array}$} & $\begin{array}{l}\text { Gene } \\
\text { ATLAS }\end{array}$ & UK Biobank & 452,264 & 66 & $\begin{array}{l}1.02 \\
(1.01- \\
1.02)\end{array}$ & $\begin{array}{l}2.32 \mathrm{E}- \\
07\end{array}$ & 0.01 & 0.05 \\
\hline & MR Base & NA & 94,402 & 49 & $\begin{array}{l}0.81 \\
(0.49- \\
1.32)\end{array}$ & 0.3907 & 0.2565 & 0.863 \\
\hline \multirow[t]{2}{*}{$\begin{array}{l}\text { Mood } \\
\text { disorder }\end{array}$} & $\begin{array}{l}\text { Gene } \\
\text { ATLAS }\end{array}$ & UK Biobank & 452,264 & 65 & $\begin{array}{l}1.01 \\
(1.00- \\
1.01)\end{array}$ & $\begin{array}{l}6.11 \mathrm{E}- \\
06\end{array}$ & 0.58 & 0.2 \\
\hline & MR Base & NA & 96,499 & 49 & $\begin{array}{l}1.23 \\
(1.03- \\
1.48)\end{array}$ & 0.02368 & 0.965 & 0.349 \\
\hline \multirow[t]{2}{*}{ Depression } & $\begin{array}{l}\text { Gene } \\
\text { ATLAS }\end{array}$ & UK Biobank & 452,264 & 66 & $\begin{array}{l}1.02 \\
(1.01- \\
1.02)\end{array}$ & $\begin{array}{l}5.29 \mathrm{E}- \\
08\end{array}$ & 0.02 & 0.07 \\
\hline & MR Base & NA & 180,866 & 49 & $\begin{array}{l}1.10 \\
(1.04- \\
1.17)\end{array}$ & $\begin{array}{l}5.97 \mathrm{E}- \\
04\end{array}$ & 7.54E-06 & 0.142 \\
\hline \multirow[t]{2}{*}{$\begin{array}{l}\text { Bipolar } \\
\text { disorder }\end{array}$} & $\begin{array}{l}\text { Gene } \\
\text { ATLAS }\end{array}$ & UK Biobank & 452,264 & 66 & $\begin{array}{l}1.00 \\
(1.00- \\
1.00)\end{array}$ & 0.19 & 0.13 & 0.66 \\
\hline & MR Base & $\begin{array}{l}\text { Bipolar } \\
\text { Disorder } \\
\text { Working } \\
\text { Group of the } \\
\text { Psychiatric } \\
\text { Genomics } \\
\text { Consortium }\end{array}$ & 51,710 & 66 & $\begin{array}{l}1.24 \\
(1.04- \\
1.49)\end{array}$ & 0.02 & 7.97E-05 & 0.122 \\
\hline $\begin{array}{l}\text { Anxiety } \\
\text { disorder }\end{array}$ & MR Base & NA & 93,291 & 49 & $\begin{array}{l}1.18 \\
(0.92- \\
1.53)\end{array}$ & 0.1951 & 0.7287 & 0.677 \\
\hline PTSD & MR Base & NA & 89,058 & 49 & $\begin{array}{l}0.77 \\
(0.33- \\
1.80)\end{array}$ & 0.5392 & 0.9251 & 0.228 \\
\hline \multirow[t]{2}{*}{ Sleep disorder } & $\begin{array}{l}\text { Gene } \\
\text { ATLAS }\end{array}$ & UK Biobank & 452,264 & 66 & $\begin{array}{l}1.00 \\
(1.00- \\
1.00)\end{array}$ & 0.28 & 0.05 & 0.78 \\
\hline & MR Base & NA & 95,720 & 49 & $\begin{array}{l}0.99 \\
(0.78- \\
1.26)\end{array}$ & 0.9221 & 0.1047 & 0.906 \\
\hline
\end{tabular}

Cl: Confidence interval; NA: Not available; OR: Odds ratio; PTSD: Post-traumatic stress disorder; SNP: Single nucleotide polymorphism 


\begin{tabular}{|c|c|c|c|c|c|c|c|c|}
\hline Outcome & $\begin{array}{l}\text { Outcome } \\
\text { source }\end{array}$ & Consortium & $\begin{array}{l}\text { Sample } \\
\text { size }\end{array}$ & $\begin{array}{l}\text { SNPs } \\
(\mathrm{N})\end{array}$ & $\begin{array}{l}\text { OR } \\
\text { (95\% } \\
\text { Cl) }\end{array}$ & $P$ value & $\begin{array}{l}\text { Heterogeneity } \\
Q \text { tests } P \\
\text { value }\end{array}$ & $\begin{array}{l}\text { MR- } \\
\text { Egger } \\
\text { intercep } \\
\text { test } P \\
\text { value }\end{array}$ \\
\hline $\begin{array}{l}\text { Eating } \\
\text { disorder }\end{array}$ & MR Base & NA & 96,499 & 49 & $\begin{array}{l}1.19 \\
(0.62- \\
2.29)\end{array}$ & 0.5919 & 0.2046 & 0.424 \\
\hline \multirow[t]{2}{*}{ Schizophrenia } & $\begin{array}{l}\text { Gene } \\
\text { ATLAS }\end{array}$ & UK Biobank & 452,264 & 66 & $\begin{array}{l}1.00 \\
(1.00- \\
1.00)\end{array}$ & 0.10 & 0.01 & 0.93 \\
\hline & MR Base & $\begin{array}{l}\text { Schizophrenia } \\
\text { Working } \\
\text { Group of the } \\
\text { Psychiatric } \\
\text { Genomics } \\
\text { Consortium }\end{array}$ & 77,096 & 66 & $\begin{array}{l}1.47 \\
(1.21- \\
1.78)\end{array}$ & $\begin{array}{l}1.04 \mathrm{E}- \\
04\end{array}$ & 3.98E-16 & 0.0455 \\
\hline
\end{tabular}

In particular, a positive association was observed between genetically predisposed trauma and the risk of mood disorder and depression in UK Biobank $(\mathrm{OR}=1.01,95 \% \mathrm{Cl}, 1.00-1.01, P=6.11 \mathrm{E}-06$; $\mathrm{OR}=1.02,95 \% \mathrm{Cl}, 1.01-1.02, P=$ $5.29 \mathrm{E}-08$; respectively), as well as in data source from $\mathrm{MR}$ Base $(\mathrm{OR}=1.23,95 \% \mathrm{Cl}, 1.03-1.48, P=0.02 ; \mathrm{OR}=1.10,95 \%$ $\mathrm{Cl}, 1.04-1.17, P=5.97 \mathrm{E}-04$; respectively). Egger intercept did not indicate the presence of horizontal pleiotropy, whereas significant heterogeneity (Cochran's $Q P<0.05$ ) was observed for trauma and depression. However, other Mendelian randomization analysis methods were not support these findings, except for weighted median. Further investigations are thus warranted to elucidate these suggestive findings.

Besides, summary statistics from MR Base showed that genetically predisposed trauma increased risk of bipolar disorder $(\mathrm{OR}=1.24,95 \% \mathrm{Cl}, 1.04-1.49, P=0.02)$ and schizophrenia $(\mathrm{OR}=1.47,95 \% \mathrm{Cl}, 1.21-1.78, P=1.04 \mathrm{E}-04)$. However, no association was observed between genetically predisposed trauma and risk of post-traumatic stress disorder, anxiety disorder, sleep disorder, and eating disorder, with no evidence of effect heterogeneity or horizontal pleiotropy.

Leave-one-out analysis suggested that no single instrument was strongly driving the overall effect of trauma on psychiatric disorder, indicating that these results were not sensitive to SNP selection. Funnel plots, scatter plots and leave-one-out plots were shown in Fig. 1, and Supplementary Fig. S1-6 online.

Furthermore, we evaluated whether the correlation between trauma and psychiatric disorder was influenced by obesity, smoking status, alcohol consumption, diabetes mellitus, vitamin D use, or physical activity. The results demonstrated that genetically predisposed trauma was not causally associated with these potential confounders and mediators except obesity class 1 [body mass index(BMI):35-39.9 kg/m²] (OR=1.24, 95\% Cl 1.07-1.43, $P=0.004)$. See Table 2. 
Table 2

Causal effects between genetically predisposed trauma and potential confounders.

\begin{tabular}{|llllll|}
\hline Trait & First author & Consortium & Sample size & OR $(95 \%$ Cl) & Pvalue \\
\hline Obesity class 1 (BMI: $30-34.9 \mathrm{~kg} / \mathrm{m} 2)$ & Berndt SI & GIANT & 98,697 & $1.24(1.07-1.43)$ & 0.004 \\
\hline Obesity class 2 (BMI: $35-39.9 \mathrm{~kg} / \mathrm{m} 2)$ & Berndt SI & GIANT & 72,546 & $1.18(0.92-1.50)$ & 0.20 \\
\hline Obesity class 3 (BMI: $\geq 40 \mathrm{~kg} / \mathrm{m} 2)$ & Berndt SI & GIANT & 50,364 & $1.31(0.87-1.97)$ & 0.20 \\
\hline Alcohol consumption & Clarke & UK Biobank & 112,117 & $0.99(0.97-1.02)$ & 0.52 \\
\hline Smoking status (ever vs never) & Neale & Neale Lab & 336,024 & $1.02(1.00-1.04)$ & 0.07 \\
\hline Physical activity & Ben Elsworth & MRC-IEU & 440,512 & $1.03(0.97-1.09)$ & 0.30 \\
\hline Vitamin D use & Ben Elsworth & MRC-IEU & 64,949 & $0.99(0.98-1.01)$ & 0.29 \\
\hline Diabetes mellitus & NA & NA & 96,499 & $1.17(0.99-1.38)$ & 0.06 \\
\hline BMI: Body mass index; Cl: Confidence interval; NA: Not available; OR: Odds ratio. & & \\
\hline
\end{tabular}

\section{Discussion}

In this study, we performed a comprehensive two-sample MR analysis using trauma-associated SNPs as genetic instruments to determine the causal role of trauma in risk of psychiatric disorder. Our study suggested that trauma exposure was causally associated with an increased risk of psychiatric disorder, which highlights the critical role of trauma exposure in shaping risk for psychopathology throughout the life course.

Mendelian randomization rests on three main assumptions ${ }^{18}$. Assumption one is that the instrumental variables must be associated with the phenotype. To satisfy the first assumption, we selected SNPs with a genome-wide significant association with trauma ${ }^{5}$. Assumption two is that the instrumental variables should not be associated with confounders. Assumption three is that the instrumental variables should affect the outcome only through the phenotype. We carried out statistical tests and sensitivity analyses to evaluate the potential violation of the second and third assumptions. Besides, we evaluated horizontal pleiotropy by MR-Egger regression method, and the results also did not show any evidence for the presence of horizontal pleiotropy for any of the reported associations.

Furthermore, potential confounders such as obesity, smoking status, alcohol consumption, diabetes mellitus, vitamin D use, and physical activity were checked in our study. The results demonstrated that genetically predisposed trauma was not causally associated with these potential confounders except obesity class 1 (BMl: $30-34.9 \mathrm{~kg} / \mathrm{m}^{2}$ ), the risk of which increased by $24 \%$. Given that the results of epidemiological studies concerning the effects of obesity on psychiatric disorder risk were conflicting ${ }^{19-21}$, this confounder was unlikely to influence the trauma-psychiatric disorder relation from our study. Nevertheless, caution is required in interpreting the outcomes.

Although trauma has been shown to play a meaningful role in the etiology of some common psychiatric disorders, the relationship between them is complex and poorly understood. Previous studies have provided evidence for underlying mechanisms through which trauma may influence mental health. Specifically, trauma exposure has been linked with HPA axis dysregulation and emotion dysregulation, which could increase risk of psychiatric disorders and transitions between disorders ${ }^{22,23}$. In addition, trauma appears to be an important correlate of a cascade of psychiatric disorders, between which both gene-environment correlations and gene-environment interactions have been observed ${ }^{5,24}$. Further studies are needed to illustrate the possible mechanisms. Interestingly, our results do not support a positive association 
between trauma and PTSD, which were inconsistent with previous observational studies ${ }^{25-27}$. Therefore, we speculate that there is only a simple epidemiological correlation, instead of a causal association between them.

Our study has several limitations. First, although the F-statistic satisfied the threshold, the selected SNPs for trauma exposure could only explain $1.79 \%$ of the phenotypic variation, other unknown trauma-related SNPs could also play an important role in the development of psychiatric disorder. Second, although sensitivity analyses have been conducted, we cannot assure that each SNP meets the three MR assumptions as instrumental variables. We checked several confounding factors such as obesity, smoking status, alcohol consumption, diabetes mellitus, vitamin D use, and physical activity. However, unknown possible confounders may inevitably affect causal inference. Third, statistical heterogeneity was observed in some estimates, which may require further investigation. Finally, the summary statistics used in our study were from participants of European ancestry, which limits the inference of findings in other populations, further studies in non-European populations are required.

\section{Conclusions}

In summary, our study suggested a causal effect of trauma on psychiatric disorder, especially mood disorder (mainly bipolar and depressive disorders). However, Mendelian randomization evidence did not support an association between trauma and risk of post-traumatic stress disorder, anxiety disorder, sleep disorder, and eating disorder. Better designed cohort studies and MR analysis are necessary to examine our findings, and deepen our understanding of their associations.

\section{Declarations}

\section{Availability of data and materials}

Summary statistics of trauma were obtained from GWAS catalog (www.ebi.ac.uk/gwas). Summary statistics of outcome were obtained from the Gene ATLAS (GeneATLAS I The University of Edinburgh) and MR-Base platform (www.mrbase.org).

\section{Competing interests}

The authors declare that they have no competing interests

\section{Funding}

This study was funded by China Postdoctoral Science Foundations (2014M562594, 2015T81090), the Military Biosafety Project (no.19SWAQ18), and Postdoctoral Science Foundation of Chongqing (Xm2015034).

\section{Authors' contributions}

G.L. and D.G. contributed to conception and design of the study; D.G. and S.O. did the literature search, data extraction and quality assessment; G.L. and D.G. did statistical analysis; D.G. wrote the first draft of the original manuscript with significant contributions from S.O. and G.L.. All authors read and approved the final manuscript.

\section{References}

1. Breslau, N., Peterson, E. L., Poisson, L. M., Schultz, L. R. \& Lucia, V. C. Estimating post-traumatic stress disorder in the community: lifetime perspective and the impact of typical traumatic events. Psychological medicine, 34, 889898 (2004). 
2. Arditte Hall, K. A., Bartlett, B. A., Iverson, K. M. \& Mitchell, K. S. Eating disorder symptoms in female veterans: The role of childhood, adult, and military trauma exposure. Psychological trauma: theory, research, practice and policy, 10, 345-351 (2018).

3. Barzilay, R. et al. Association between traumatic stress load, psychopathology, and cognition in the Philadelphia Neurodevelopmental Cohort. Psychological medicine, 49, 325-334 (2019).

4. Brown, R. C. et al. Trauma Exposure and Axis I Psychopathology: A Co-twin Control Analysis in Norwegian Young Adults. Psychological trauma: theory, research, practice and policy, 6, 652-660 (2014).

5. Coleman, J. R. I. et al. Genome-wide gene-environment analyses of major depressive disorder and reported lifetime traumatic experiences in UK Biobank. Mol Psychiatry, 25, 1430-1446 (2020).

6. Mysliwiec, V. et al. Trauma associated sleep disorder: A parasomnia induced by trauma. Sleep medicine reviews, 37, 94-104 (2018).

7. Popovic, D. et al. Childhood Trauma in Schizophrenia: Current Findings and Research Perspectives. Frontiers in neuroscience, 13, 274 (2019).

8. Fewell, Z., Davey Smith, G. \& Sterne, J. A. The impact of residual and unmeasured confounding in epidemiologic studies: a simulation study. Am J Epidemiol, 166, 646-655 (2007).

9. Smith, G. D. \& Ebrahim, S. 'Mendelian randomization': can genetic epidemiology contribute to understanding environmental determinants of disease? Int J Epidemiol, 32, 1-22 (2003).

10. Davies, N. M., Holmes, M. V. \& Davey Smith, G. Reading Mendelian randomisation studies: a guide, glossary, and checklist for clinicians. BMJ, 362, k601 (2018).

11. Lyons, M. J. et al. Do genes influence exposure to trauma? A twin study of combat. American journal of medical genetics, 48, 22-27 (1993).

12. Stein, M. B., Jang, K. L., Taylor, S., Vernon, P. A. \& Livesley, W. J. Genetic and environmental influences on trauma exposure and posttraumatic stress disorder symptoms: a twin study. The American journal of psychiatry, 159, 1675-1681 (2002).

13. Power, R. A. et al. Estimating the heritability of reporting stressful life events captured by common genetic variants. Psychological medicine, 43, 1965-1971 (2013).

14. Yarmolinsky, J. et al. Circulating Selenium and Prostate Cancer Risk: A Mendelian Randomization Analysis. J Natl Cancer Inst, 110, 1035-1038 (2018).

15. Palmer, T. M. et al. Using multiple genetic variants as instrumental variables for modifiable risk factors. Stat Methods Med Res, 21, 223-242 (2012).

16. Bowden, J. \& Holmes, M. V. Meta-analysis and Mendelian randomization: A review. Res Synth Methods, 10, 486496 (2019).

17. Bowden, J., Davey Smith, G. \& Burgess, S. Mendelian randomization with invalid instruments: effect estimation and bias detection through Egger regression. Int J Epidemiol, 44, 512-525 (2015).

18. Glymour, M. M., Tchetgen, T., Robins, J. M. \& E. J. \& Credible Mendelian randomization studies: approaches for evaluating the instrumental variable assumptions. Am J Epidemiol, 175, 332-339 (2012).

19. de Wit, L. et al. Depression and obesity: a meta-analysis of community-based studies. Psychiatry research, 178, 230-235 (2010).

20. Hach, I. et al. Obesity and the risk for mental disorders in a representative German adult sample. European journal of public health, 17, 297-305 (2007).

21. John, U., Meyer, C., Rumpf, H. J. \& Hapke, U. Relationships of psychiatric disorders with overweight and obesity in an adult general population. Obesity research, 13, 101-109 (2005). 
22. Trickett, P. K., Noll, J. G., Susman, E. J., Shenk, C. E. \& Putnam, F. W. Attenuation of cortisol across development for victims of sexual abuse. Development and psychopathology, 22, 165-175 (2010).

23. Ehring, T. \& Quack, D. Emotion regulation difficulties in trauma survivors: the role of trauma type and PTSD symptom severity. Behavior therapy, 41, 587-598 (2010).

24. Walsh, K., McLaughlin, K. A., Hamilton, A. \& Keyes, K. M. Trauma exposure, incident psychiatric disorders, and disorder transitions in a longitudinal population representative sample. Journal of psychiatric research, 92, 212218 (2017).

25. Wolff, N., Huening, J., Shi, J. \& Frueh, B. C. Trauma exposure and posttraumatic stress disorder among incarcerated men. Journal of urban health: bulletin of the New York Academy of Medicine, 91, 707-719 (2014).

26. Jowett, S., Karatzias, T. \& Albert, I. Multiple and interpersonal trauma are risk factors for both post-traumatic stress disorder and borderline personality disorder: A systematic review on the traumatic backgrounds and clinical characteristics of comorbid post-traumatic stress disorder/borderline personality disorder groups versus singledisorder groups. Psychology and psychotherapy, 93, 621-638 (2020).

27. Kessler, R. C. et al. Trauma and PTSD in the WHO World Mental Health Surveys. European journal of psychotraumatology, 8, 1353383 (2017).

\section{Figures}




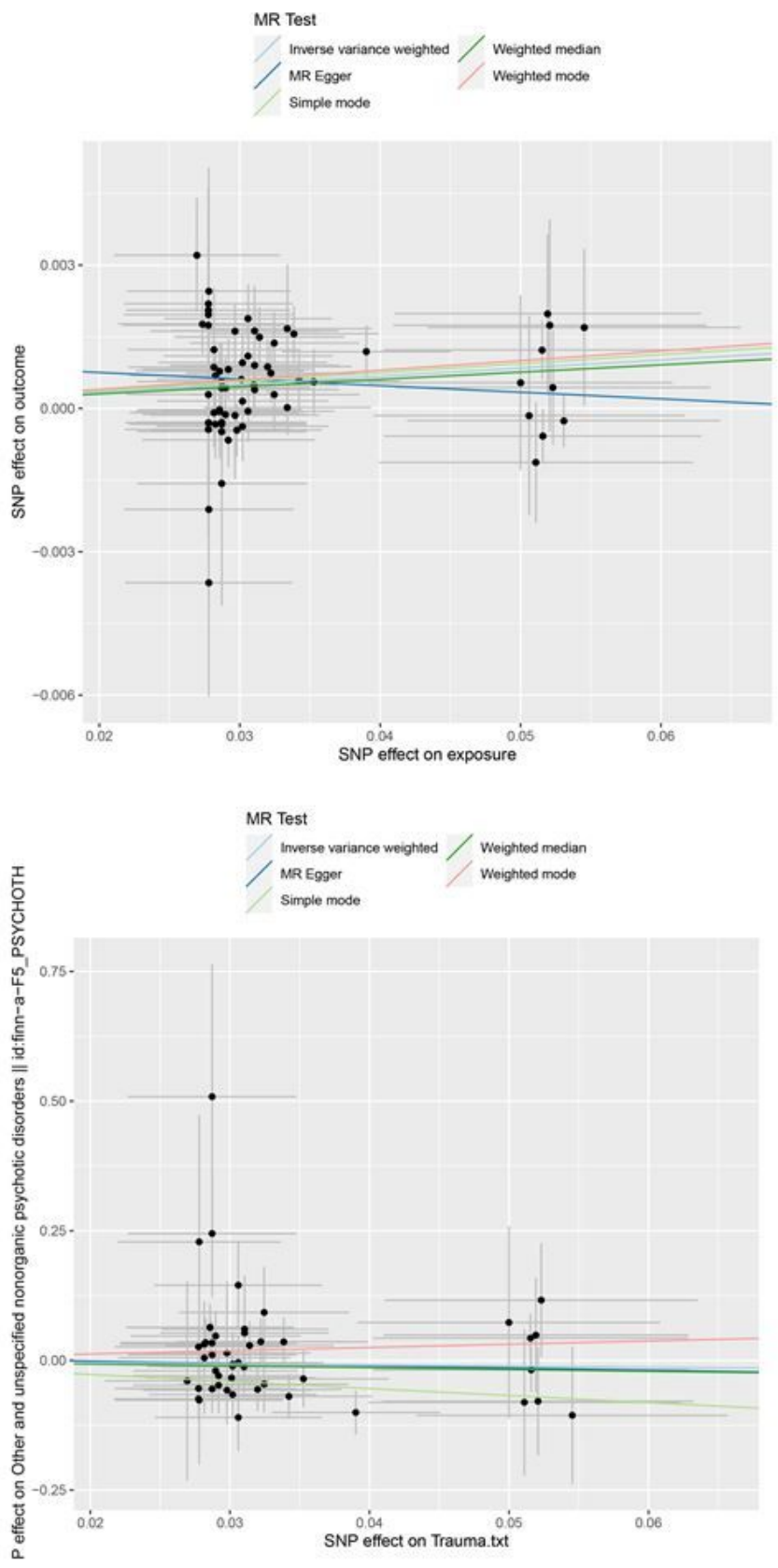

\section{Figure 1}

Scatter plots of the genetic causal associations between trauma and psychiatric disorder using different MR methods. (A) Trauma and psychiatric disorder in UK Biobank; (B) trauma and psychiatric disorder in MR Base. The slopes of the line represent the causal association for different methods. The light green line represents the simple mode estimate, the dark green line represents the weighted median estimate, the pink line represents the weighted mode estimate, the light blue line represents the inverse variance weighted estimate, and the dark blue line represents the MR Egger.

\section{Supplementary Files}

This is a list of supplementary files associated with this preprint. Click to download. 
- Supplementarymaterials.docx

Page $12 / 12$ 